\title{
Ontology-based Demand-Side Flexibility Management in Smart Grids using a Multi-Agent System
}

\author{
J.L. Hippolyte, S. Howell, B. Yuce, M. Mourshed \\ BRE Trust Centre for Sustainable Engineering \\ School of Engineering, Cardiff University \\ Cardiff, United Kingdom \\ hippolytej@cardiff.ac.uk
}

\author{
H. A. Sleiman, M. Vinyals, L. Vanhee \\ CEA, LIST, LADIS \\ 91191, Gif Sur Yvette, France \\ hassan.sleiman@cea.fr
}

\begin{abstract}
Power distribution network management must integrate with demand side management, alongside distributed energy resources, in order to meet sustainability, resilience, and economic challenges through a smart grid approach. This paper presents an implementation of the Universal Smart Energy Framework (USEF) through a multiagent system and a novel semantic web ontology, which aligns and enriches relevant existing standards. USEF provides a common specification of the market processes and information exchange but does not specify the internal reasoning of the different roles involved. The authors explain the systematic design and development process from the requirements of the energy-flexibility value chain to software implementation. The underpinning ontology formalizes a domain perspective which is coherent with existing standards, and is sufficient for the agent-oriented implementation of the mentioned framework. As well as contributing this model as a web ontology artifact, the presented work utilizes metaprogramming to transform the domain model into a standard agent communication language ontology. The research reported in this paper is expected to lead towards efficient and scalable development of decision support and automation software for smart grids.
\end{abstract}

Key words-smart grid, demand response, multi-agent system, ontology, flexible energy market.

\section{INTRODUCTION}

Future energy markets, in which most traditional consumers will be replaced by prosumers (a new type of energy user acting as both a consumer and a producer) will bring about new challenges for the cost-effective management of the grid [1]. Since traditional electricity grids are not inherently adaptive and may not respond well to the periods of peak demand, electricity markets need to evolve towards a usercentric grid [2]. Energy suppliers will have to balance their own generation while taking into consideration the customers demand response assets [3]. A mutual benefit can be achieved by leveraging the flexibility of demand response equipment by minimizing balancing costs at the supply level and achieving up to zero net energy consumption at the customer level. Implementing flexibility across the energy system requires a complete rethink of the distribution network and system management. Mourshed et al. [4] have identified the four strategic challenges that condition the success of demandresponsive smart grids: interoperability, decentralized selforganizing architecture, reliability and security, and innovative business models. This paper aims to provide an efficient solution to the integration of Information and communications technology (ICT) with energy infrastructures, particularly with regard to the two first challenges. The Universal Smart Energy Framework (USEF) [3] provides a coherent framework of standards to allow seamless energy and flexibility value chains, from prosumers to the transmission network. The intrinsic distributed nature of the energy and flexibility value chains makes them desirable candidates for a multi-agent system (MAS) implementation. The core of the USEF specifications is the market-based coordination mechanism and the processes governing it that define how the different active stakeholders should behave and interact. Although the USEF provides a common specification of the market processes and information exchange, it does not specify how the different roles implement the required functions to participate in such processes (e.g. USEF does not specify how an aggregator optimizes its portfolio and determines how much flexibility available and when). Defining USEF-compliant fully functional implementations is therefore an open issue. Moreover, as illustrated in Fig. 1, USEF does not itself define the standards that MAS need to model the multi-scale flexible energy domain and data and interoperate with smart energy infrastructures. This paper aims to formalize a reconciliation of relevant standards, framed by the flexibility and energy supply value chains as defined by USEF, into a semantic web ontology (called MAS2TERING ${ }^{1}$ ontology) in order to facilitate the domain capture and interoperability of agents for smart grid management.

This background has motivated the authors to carry out research on the best approach for decision support/control software developers to produce an efficient, semantically aware and operationally accurate software environment for managing flexibility in urban power distribution networks. Sect. II presents the semantic resources and methods on the basis of

\footnotetext{
${ }^{1}$ After the eponymous project MAS2TERING http://www.mas2tering.eu.
} 


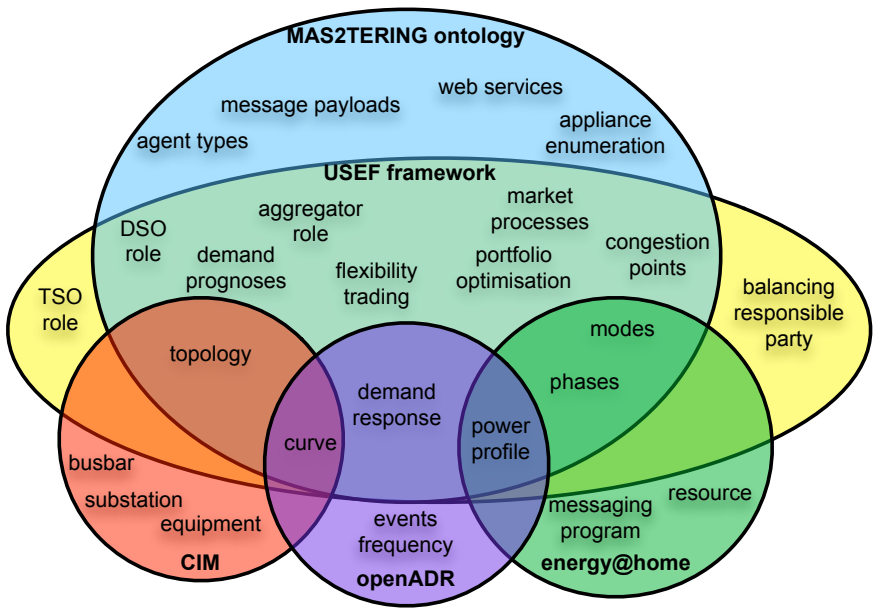

Fig. 1. Venn diagram illustrating some of the common and distinct concepts across key standards and the MAS2TERING ontology.

which the authors developed their data model and domain ontology. Sect. III gives an overview of the models produced in accordance with the Gaia methodology [5], including roles, behaviors, services, acquaintances, interactions and domain data model. Finally, Sect. III-D summarizes the process followed by the authors during the implementation phase.

\section{BACKGROUND}

\section{A. Energy distribution communication \& data models}

Open communication between smart devices using common protocols is crucial to interoperability. The IEEE standards committee identified the challenge of interoperable protocols, data formats and meaning [6]. The use of a common vocabulary and data model mitigates the effort required for software artefacts to communicate effectively with others in the energy management system [7]. Significant steps have been taken towards semantic modeling of the energy domain, including the demand side management (DSM) sub-domain. Arguably, the most widely noted example is IEC 61970 Common Information Model (CIM) [8], and its distribution management extension IEC 61968 [9], although the Open Automated Demand Response (OpenADR) [10] and energy@home [11] models are also highly relevant. However, as shown in Fig. 1, none of these standards cover the whole semantics involved in a flexible urban energy network on its own, and they are not formally aligned with each other.

The physical transport of energy underlies the flexibility and energy supply value chains modeled by the USEF framework. As García et al. [12] stated, a smart grid is a network of networks, which requires advanced communication protocols and standards such as Home Area Networks (HANs), Building Energy Management Systems (BEMS), Advanced Metering Infrastructure Networks (AMINs), Neighborhood Area Networks (NANs) and so on. Sharing knowledge about physical system integration, organization procedures and standards is key to complex cyber-physical systems, such as smart grids.
Gillani et al [13] proposed that a detailed knowledge integration and organization can be achieved through an ontologybased approach. Ontologies have been successful in integrating the knowledge required for solving complex problems such as energy management problems [14]. Further, the usage of ontologies for interoperability also provides the capability of dealing with multi-scale control and integration [15].

The conjunction of the lack of software interoperability in the current energy system with the need of integrating largescale flexible demand response and prosumers into the future energy system has motivated the emergence of new energy frameworks. One of the most promising is USEF [16], a reference framework for market design, actor interactions and common flexibility services between the actors. The USEF foundation promotes an open framework of specifications, designs, and implementation guidelines for the realization of a smart energy system. USEF covers the following features of such systems:

- interaction model,

- market-based coordination mechanisms,

- grid operations,

- smart energy services,

- privacy and security guideline,

- and IT architecture.

USEF defines the different stakeholders and their roles and responsibilities within both the energy supply value chain and the flexibility value chain, and it facilitates the mapping of these roles with current or emerging energy commodities and business models.

Furthermore, it ensures that the value of flexibility can be maximized and transferred. USEF proposes the aggregator as the center of the flexibility value chain in smart grids, whose services are provided to the Prosumer, the Balance Responsible Party (BRP), The Distribution System Operator (DSO), and the Transmission System Operator (TSO). In this paper, the authors focus on the Prosumer, Aggregator, and the DSO, for which the authors only consider the role of congestion/capacity management.

The CIM is a 3-layer broad domain model that aims to facilitate power management processes such as outage management, asset management and customer information management. The CIM is arguably not well suited to DSM due to its lack of modeling at the last mile of the supply chain.

The OpenADR conceptualizes demand response (a subset of DSM) through a data and communication specification. Even though it formalizes concepts between the consumer and the supplier of energy, such as market context, dynamic pricing and event descriptions, it fails to integrate data and commands at the device level.

The energy@home data model specifies a representation model for home area networks, including smart appliances, power profiles, renewable energy generation, smart meters and smart user interfaces. It is based on the CIM approach and is broadly aligned with the OpenADR schema. 


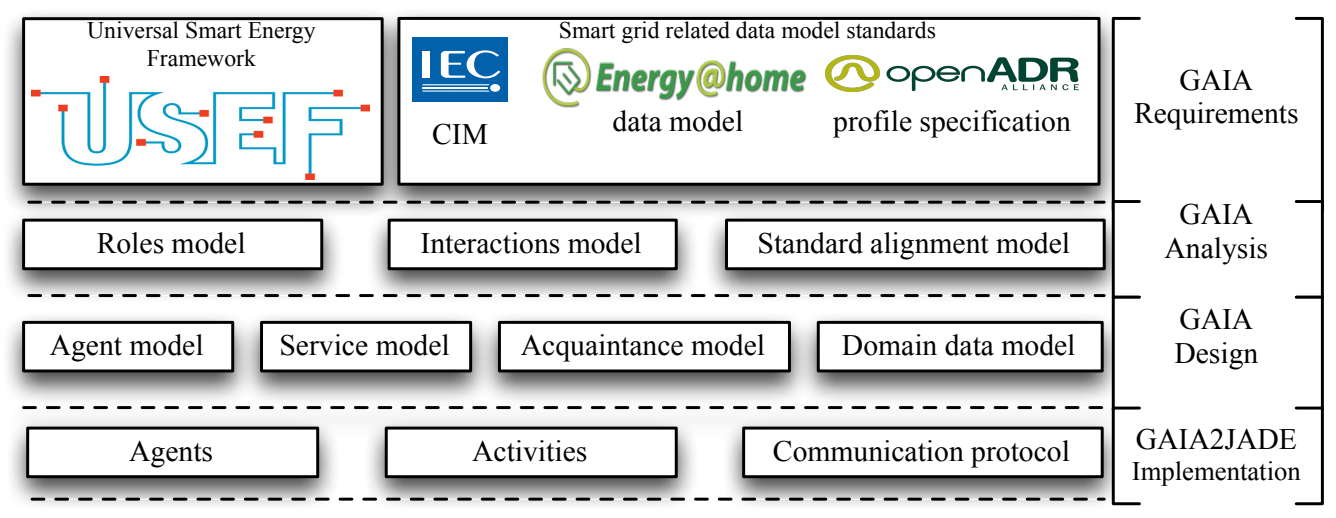

Fig. 2. Overall methodology, from requirements to implementation.

The union of USEF, CIM, openADR and energy@home should represent the scope required for the delivery of DSM across the supply chain from generation to consumer appliances. However, the energy and flexibility value chains inherently incorporates widely varied domain perspectives, manifesting as inconsistencies, misalignments, and incompatibilities between existing standards such as CIM, openADR, and energy@home. For example, the term equipment could refer to transmission system equipment, or domestic appliance equipment; this lack of explicitness could lead to misunderstood messages between software, and incorrect property assignments. In order to overcome this, a formalized domain perspective which is agreed upon by experts in the demand side management and distributed energy resource fields would be highly beneficial. This would provide a reference meaning for terms, expressed in a rich, machine interpretable manner. Further, the use of ontological constructs allows a highly expressive means of achieving this, mitigating the potential for misunderstanding or incompatibility between virtual entities.

Towards this, the presented work contributes a coherent manifestation of such an ontology, which incorporates standards relevant to the USEF framework, as well as novel concepts from the USEF framework, and further concepts deemed necessary for implementing the USEF approach through a MAS, as shown in Fig. 1. This candidate ontology benefits from its alignment of existing standards, such in as the reuse of the energy@ home power profile modeling pattern; existing energy@home smart appliances could join flexibility markets with minimal effort within the proposed implementation. Further, the synchronized use of the OWL 2 Web Ontology Language $^{2}$ (simply referred to as OWL in the rest of this paper) and JADE Java bean ontologies allows integration of the agents with web services through a semantic web approach, and provides greater extensibility for further web services. The candidate ontology is presented in Sect. III-C below.

${ }^{2}$ https://www.w3.org/TR/owl2-syntax

\section{B. The Gaia and Gaia2JADE methodologies}

Multiagent Systems (MAS) have become popular solutions to tackle the complexity of decentralized systems [17]. In a multi-agent approach, each component (physical or abstract) of a system is autonomous and can interact or communicate with its environment and with other agents via predefined interfaces and protocols. MAS have proven to bring together many disciplines in an effort to build distributed, intelligent, and robust applications [18], especially for smart grid solutions. A number of prominent agent-oriented design methodologies have been proposed in the literature and applied by practitioners [19]. The Gaia methodology [5] provides methodological tools towards successfully and efficiently implementing problem-solving MASs. The first phase of Gaia is the analysis, which extracts from the system requirements: (a) the roles of the organization (including an informal description, permissions, activities and protocols to be performed by the role) and (b) the interactions that should be conducted (including the purpose, the initiator, the responder, inputs, outputs and processing to be performed by the interaction). The output of the analysis phase is then used towards producing more concrete artifacts in the design phase, which further describes the agents (types of agents in the system), services (activities to be performed by a role) and acquaintances (describing who is connected to whom), as pictured in the design and analysis layers of Fig. 2. Gaia2JADE complements the implementationindependent Gaia methodology to support MAS development using the JAVA Agent DEvelopment Framework (JADE) [20]. It adds an additional phase that follows GAIAs design phase, called JADE implementation. The JADE implementation phase provides MAS developers with systematic steps and guidelines to produce the agents Java code and a repository communication protocols, the implementation of the activities, and agent behaviors.The application of Gaia and Gaia2JADE methodologies is detailed in Sect. III-D below.

\section{Ontologies and multiagent systems}

Researchers and practitioners have used OWL ontologies in conjunction or as a complement of MAS for various usages. 
Neruda [21] presented a logical formalism to describe computational agents and MAS, along with a practical implementation using OWL-DL, a reasoner and JADE, for the purpose of automatic configuration of MAS. An application of this approach has been the deployment of a meta-learning system able to assist experts in configuring data mining processes [22]. Using ontologies in order to support the behavioral pattern of the agents is not in the scope of this paper.

The use of ontology in this paper is more similar in nature to other research that has focused on using ontologies to facilitate the development of "domain-specific agent-oriented reusable software artifacts" [23]. Girardi and Lindoso, for example, created an ontology-based methodology called Multi-Agent Domain Engineering (MADEM) to that effect. An alternative to MADEM has been proposed by Bittencourt et al. based on Gaia [24]. These authors implemented OWL ontologies of both Gaia and JADE, as well as a set of SWRL (Semantic Web Rule Language) in order to map the Gaia models with JADE behaviors.

The semi-automated approach described in this paper makes use of an OWL ontology to cope with the heterogeneity, changeability and cyber-physical nature of the end application of the targeted MAS. In practice, the domain experts edit/reconcile existing (and ideally authoritative) ontological resources using the Protégé ${ }^{3}$ tool and then use a dedicated Eclipse plugin to automatically generate the JADE-compliant Java classes that capture the domain data model, as described in Sect. III-C.

\section{MAS DESIGN AND IMPLEMENTATION}

Gaia is particularly suitable regarding the goals of the authors: GAIA's purpose is building large-scale real-world MASs and USEF specifications can simply be mapped to Gaia design requirement. The authors consequently established a systematic correspondence between the actors, roles and value chain defined in the market organization model of USEF and the agents, roles and behaviors resulting in an MAS [20].

\section{A. Gaia models}

1) Roles model: The Roles model identifies the detected roles based on the requirements. The authors identified four roles in the system, namely: device, Customer Energy Management System (CEMS), aggregator (AGGR), and the DSO. These roles match the USEF, expanded with a role for managing a house (CEMS) and another for a device in a house, as depicted in Fig.3, where micro-generation, appliance and battery are examples of device roles. The device role is responsible for managing an energy-consuming device; i.e., it is responsible for managing the interaction with the user, to operate the device, to inform CEMS about device flexibilities and consumption and handles control requests from the CEMS. This role is allowed to operate the device, interact with the user and CEMSs. The CEMS role is responsible for minimizing the energy bill of the house matching the configuration defined

\footnotetext{
${ }^{3}$ Stanford's open source OWL editor available at http://protege.stanford.edu
}

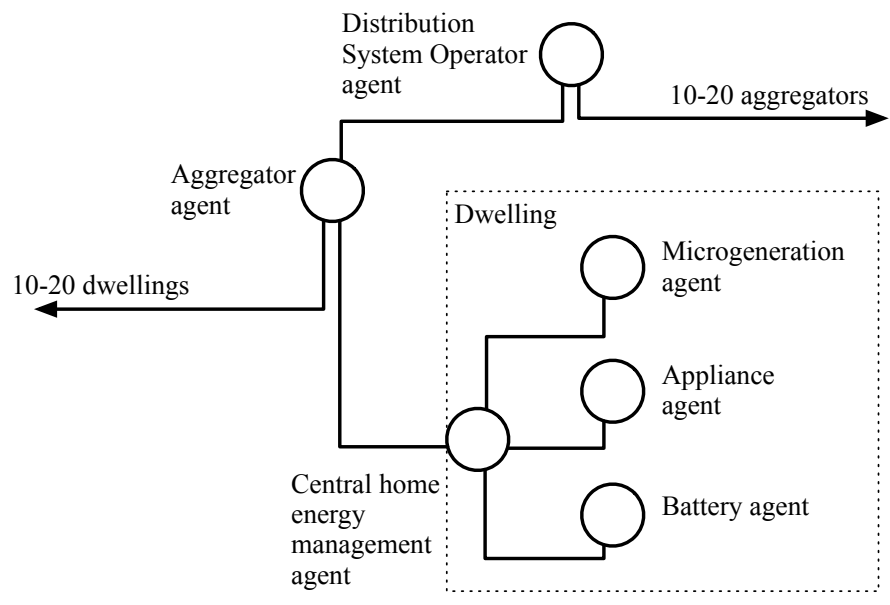

Fig. 3. Multi-agent roles schematized.

by the prosumer. More precisely, this role is responsible for locally optimizing energy consumption and for bargaining flexibility with the AGGR. The agent is allowed to interact with the AGGR and the in-house devices. The AGGR role helps to lower the energy costs of the set of CEMS it monitors in two ways: by locally optimizing the consumption of the monitored CEMS in enabling a local flexibility market, and by being a mediator between CEMS and DSOs for trading flexibility. More precisely, this role is responsible for monitoring possible congestion points raised by the DSO: given a congestion point, the AGGR requests the consumption plans from CEMS and it indicates the expected global consumption to the DSO: if a congestion is expected, the AGGR tries to sell flexibility, bought from the CEMS or other AGGRs, to the DSO. In addition, the AGGR is responsible for monitoring the consumption of monitored CEMS and indicate if a deviation occurs regarding a congestion point. The DSO role is responsible for preventing congestion. More precisely, this role is responsible for predicting the future occurrence of congestion points; requesting the predicted consumption at these points from AGGRs; if congestion is expected at a point, reducing the consumed energy by buying flexibility to AGGRs.

2) Agents model: The Agent model identifies the agent types that will make up the system, and the agent instances that will be instantiated. In the present case, each role corresponds to an agent type. Thus, the authors have four types of agents: DSO, CEMS, AGGR, and Device. This classification considers that the Device agent is extended for each type of device (i.e. deferrable load, fixed load, storage unit, etc...). At the district level, there is a single DSO, whereas the other types have a cardinality higher or equal to 1 .

3) Services model: The Service model identifies the main services that are required to realize the agents role. The services are derived from the activities and protocols of the roles. The CEMS provides three services: reading and aggregating the plans from the devices, sending the P-Plan to the aggregator, and informing control signal to the devices. The AGGR also provides services: collecting and aggregating 
the plans from the CEMS.

TABLE I

ACQUAINTANCES MODEL

\begin{tabular}{|c|c|c|c|c|}
\hline & CEMS & AGGR & DSO & Device \\
\hline CEMS & & $\bullet$ & & $\bullet$ \\
\hline AGGR & $\bullet$ & $\bullet$ & $\bullet$ & \\
\hline DSO & & $\bullet$ & & \\
\hline Device & $\bullet$ & & & \\
\hline
\end{tabular}

4) Acquaintances model: The Acquaintance model documents the lines of communication between the different agents and can be used for identifying potential communication bottlenecks that may arise at runtime. This model provides a high-level overview of the origins of bottlenecks, skipping low-level details that are less relevant at this stage of the design phase (e.g. details about the content of messages).

\section{B. Interaction models}

This model depicts the interactions that agents can initiate with each other. Based on USEF, the authors identified the following interactions:

- SubscribeFlexibility takes place between the CEMS and the Device agents; the CEMS interacts with the device in order to be informed about its flexibilities and further flexibility changes.

- InformControlSignals takes place between the CEMS and the Device agents; the CEMS provides activation instructions to the device.

- SubscribePPlan takes place between the AGGR and the CEMS agents; the AGGR requests the CEMS to send its predicted consumption for the following day (PPlan).

- OptimiseInternalPortfolio takes place between the AGGR and the CEMS; the AGGR enables the local flexibility market, enabling CEMS to negotiate flexibility with each other.

- TradeFlexibilityForPortfolioOptimisation takes place between the AGGR agents: the AGGRs initiate flexibility bargaining with one another. Possibly, this protocol can involve flexibility bargaining with CEMS.

- QueryCongestionPoints takes place between the AGGR and the DSO; the AGGR retrieves the list of congestion points it is related to and corresponding DSOs.

- QueryActiveAggregators takes place between the DSO and the AGGR; the DSO retrieves the set of active aggregators related to one of their congestion points.

- ReceiveDPrognoses takes place between the AGGR and the DSO agent; the AGGR informs the DSO about the expected consumption for a long-term congestion point.

- FlexibilityTradingAGRDSO takes place between the DSO and the AGGR; the DSO initiates flexibility bargaining with an AGGR in order to prevent congestion.

\section{Domain data model}

An ontology that encompasses most of the domain semantics required by the USEF flexibility value chain and based on the standards mentioned in Sect. II-A, has been developed for the management of domestic flexibility markets [25]. These allow consumers to sell the deferment and curtailment of their loads to a distribution service operator, through a flexible hierarchy of aggregation, and close integration with smart appliances and DERs.

Load flexibility is here defined as a market commodity of utilized peak load reduction through optional deferment and/or curtailment of consumer demand, expressed as a unit of energy.

Deferment is the shifting of a load to a time more favorable to the network operator, where the amount of flexibility is equal to the amount of energy shifted. In this way, the extent of the shift is independent to the flexibility, as the consumer sets a deadline for the task completion. This is represented in Fig. 4b, where $Q_{t o t}$ is the total energy consumption of the task, $Q_{f}$ is the flexibility utilized, $t_{0}$ is the earliest start time of the task, $t_{1}$ is the task completion deadline, and $T_{\min }$ is the minimum amount of time the task requires to be completed. Curtailment of load is then the supply of a quantity of energy over time which is less than the desired quantity. The flexibility is then the difference between the desired quantity and the supplied quantity, again expressed as an amount of energy. This is shown in Fig. 4c, where $t_{0}$ is the earliest start time of the task, $t_{1}$ is the non-negotiable deadline of the task, $Q_{f}$ is the amount of flexibility utilised, and $P_{\min }$ is the minimum amount of energy to be supplied (such as when a heating device must meet a minimum room temperature). Based on the use case analyses and the definitions of flexibility presented, devices were then categorized according to their likely flexibilities and types of variability.

Alignments with the aforementioned existing standards were formalized as OWL annotations for trivial schema conversion through a SPARQL CONSTRUCT query, or to produce an owl:equivalentClass instantiation. Whilst it would be incorrect to state that this represents full compliance or alignment with the standard, it demonstrates broad coherence with the domain perspectives of the existing standards, and paves the way for genuine compliance if the existing standards are developed into full semantic models in the future.

The domain data model, which aligns existing standards specified in various languages (UML, XML, RDF/XML) presented in Sect. III-C was expressed as an OWL ontology in an ad hoc manner. In order to utilize the ontology to formalize the semantics of the payloads encoded thanks to the Agent Communication Language proposed by the Foundation for Intelligent Physical Agents (FIPA-ACL), the candidate OWL ontology was converted into a set of JADE concept and predicate bean classes. Browsing the OWL ontology while manually writing the source code that implements the JADE bean ontology is a highly conceptually redundant task that can be handled by automatic code generation [26]. Code generation provides robustness to the development process in the early stages, while the OWL ontology is being matured by domain modelers. The model transformation has been automated by combining the open source Java framework 


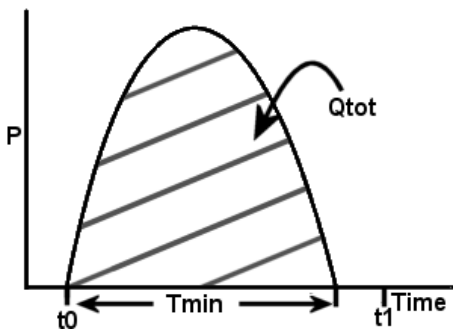

(a)

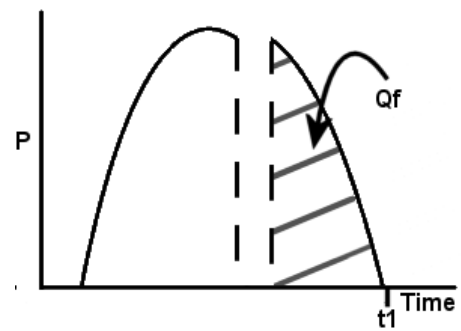

(b)

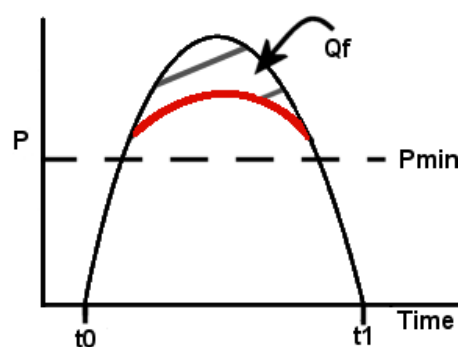

(c)

Fig. 4. Demand profiles without flexibility (a), with deferment (b), with curtailment (c).

for Semantic Web applications Apache Jena ${ }^{4}$ (to interpret the OWL file expressing the candidate ontology) and the Eclipse Java Development Tools core 5 infrastructure (to manipulate Java source code).Through this conversion process, the ontologys axioms were formalized using JADE constructs: classes (including inheritance and mapping with concepts and agent action in JADE), datatype properties (using an arbitrary correspondence between XML datatypes and Java datatypes) and object properties (referred to as predicates in JADE).

\section{Implementation phases using GAIA2Jade methodology}

In order to complete the development of the agent-based software for energy management, the authors followed the Gaia2JADE process [20], which covers the whole software development lifecycle. The authors do not provide the communication protocols and the refinement of the activities since they can be achieved straightforwardly using JADE, since the communication schema is based on JADEs communication protocols and FIPA-compliant messages [27].

The Defining JADEs behaviors of the Gaia2JADE process consists in translating the Gaia responsibilities, activities and protocols from the role model into JADE behaviors. As a brief sketch of the translation, final (lower-level) Gaia activities or protocols are represented by simple JADE behaviors. Higherlevel Gaia activities and protocols (those combining multiple activities and protocols using the Gaia Finite-State-Machinelike operators) are represented by JADE composite behaviors (e.g. FSMbehaviors).

The Define JADE agents step of the GAIA2Jade process consists in designing the agent classes. One JADE agent is to be designed per Gaia agent. Then, the agent is to be given an adequate data model, as defined in Sect. III-C in its memory and the adequate behavior in its setup function. The agent types are DSO, AGGR agent, CEMS agent, and Device Agent. The latter can be extended and specified for each device type, whereas all the agents extend JADEs class Agent.

\section{Discussion \& CONCLUSION}

This paper presents a USEF-compliant implementation based on a multi-agent system (MAS). In more detail, the

\footnotetext{
${ }^{4} \mathrm{http}: / /$ jena.apache.org

${ }^{5}$ https://www.eclipse.org/jdt/core/
}

proposed implementation uses multi-agent negotiation mechanisms to realize the USEF market-based coordination process in which a large number of stakeholders must agree on how to optimality divide the available flexibility over the different services at each period in time. In addition to this, this paper contributes to formalize a reconciliation of standards relevant to demand-responsive smart grids, framed by the flexibility and energy supply value chains as defined by USEF, into a semantic web ontology in order to facilitate the domain capture and interoperability of MAS for smart grid management. In order to achieve these objectives, a candidate ontology that covers the semantics of the whole flexibility value chain, from the DSO level to the device level, is proposed and the Gaia and Gaia2JADE methodologies were applied, taking into account the requirements by USEF. The resulting MAS, is able to model the data coming from the physical and business components of smart grids and reason over these USEF compliant concepts. This MAS and its transparently synchronized domain semantic web ontology, not only enable efficient decision support in emergent demand-response aware energy distribution networks but could also balance edgecloud processing distribution for security and scalability thanks to a close integration of web services and agents. With the behavioral design of a USEF-enabled agent architecture and the implementation of the the domain data model metaprogram being done, future works will include deployment in test bed smart grids in order to assess the viability of the resulting MAS as an enabler of market-based flexibility.

\section{ACKNOWLEDGMENT}

The authors would like to acknowledge the financial support of the European Commission in the context of the MAS2TERING project (Ref: 619682) funded under the ICT2013.6.1 - Smart Energy Grids program.

\section{REFERENCES}

[1] S. D. Ramchurn, P. Vytelingum, A. Rogers, and N. R. Jennings, "Putting the 'smarts' into the smart grid: a grand challenge for artificial intelligence," Communications of the $A C M$, vol. 55, no. 4, p. 86, Apr. 2012. [Online]. Available: http://dl.acm.org/citation.cfm?doid=2133806.2133825

[2] Europische Kommission, Ed., European technology platform SmartGrids: vision and strategy for Europe's electricity networks of the future, ser. EUR. Luxembourg: Office for Official Publications of the European Communities, 2006, no. 22040. 
[3] U. Foundation, "USEF: THE FRAMEWORK SPECIFICATIONS 2015," Feb. 2015. [Online]. Available: http://www.usef.info/Framework/Download-the-framework.aspx

[4] M. Mourshed, S. Robert, A. Ranalli, T. Messervey, D. Reforgiato, R. Contreau, A. Becue, K. Quinn, Y. Rezgui, and Z. Lennard, "Smart Grid Futures: Perspectives on the Integration of Energy and ICT Services," Energy Procedia, vol. 75, pp. 1132-1137, Aug. 2015. [Online]. Available: http://linkinghub.elsevier.com/retrieve/pii/S1876610215012990

[5] M. Wooldridge, N. R. Jennings, and D. Kinny, "The Gaia Methodology for Agent-Oriented Analysis and Design," Autonomous Agents and Multi-Agent Systems, vol. 3, no. 3, pp. 285-312, 2000. [Online]. Available: http://dx.doi.org/10.1023/A:1010071910869

[6] IEEE Standards Committee, P. IEEE Standards Coordinating Committee 21 on Fuel Cells, Dispersed Generation and Energy Storage, Institute of Electrical and Electronics Engineers, and IEEE-SA Standards Board, IEEE guide for smart grid interoperability of energy technology and information technology operation with the electric power system (EPS), end-use applications and loads. New York, N.Y.: Institute of Electrical and Electronics Engineers, 2011. [Online]. Available: http://ieeexplore.ieee.org/servlet/opac?punumber $=6018237$

[7] K. van Dam and J. Keirstead, "Re-use of an ontology for modelling urban energy systems," in Infrastructure Systems and Services: Next Generation Infrastructure Systems for Eco-Cities (INFRA), 2010 Third International Conference on, Nov. 2010, pp. 1-6.

[8] IEC, "Energy management system application program interface (EMSAPI) Part 301: Common information model (CIM) base," 2011.

[9] "IEC 61968: Common Information Model (CIM) / Distribution Management," 2003.

[10] O. Alliance, "OpenADR 2.0 Profile Specification A Profile," 2011. [Online]. Available: http://www.openadr.org/specification

[11] E. Association, "Energy@ @ome Technical Specification," Oct. 2015. [Online]. Available: http://www.energy-home.it

[12] A. P. Garcia, J. Oliver, and D. Gosch, "An intelligent agentbased distributed architecture for Smart-Grid integrated network management." IEEE, Oct. 2010, pp. 1013-1018. [Online]. Available: http://ieeexplore.ieee.org/lpdocs/epic03/wrapper.htm?arnumber=5735673

[13] S. Gillani, G. Picard, and F. Laforest, "IntelSCEP: Towards an Intelligent Semantic Complex Event Processing Framework for Prosumer-Oriented SmartGrid.” ACM Press, 2014, pp. 1-2. [Online]. Available: http://dl.acm.org/citation.cfm?doid=2637064.2637110

[14] B. Yuce and Y. Rezgui, "An ANN-GA Semantic Rule-Based System to Reduce the Gap Between Predicted and Actual Energy Consumption in Buildings," IEEE Transactions on Automation Science and Engineering, pp. 1-13, 2015. [Online]. Available: http://ieeexplore.ieee.org/lpdocs/epic03/wrapper.htm?arnumber=7317804

[15] Q. Zhou, S. Natarajan, Y. Simmhan, and V. Prasanna, "Semantic Information Modeling for Emerging Applications in Smart Grid.” IEEE, Apr. 2012, pp. 775-782. [Online]. Available: http://ieeexplore.ieee.org/lpdocs/epic03/wrapper.htm?arnumber=6209085
[16] Frits Bliek, Arnoud Backers, Milo Broekmans, Claire Groosman, Hans de Heer, Marten van der Laan, Marijn de Koning, Jan Nijtmans, Phuong Nuygen, Tjerk Sanberg, Berry Staring, Marcel Volkerts, and Eric Woittiez, "An introduction to the Universal Smart Energy Framework," 2014. [Online]. Available: http://dx.doi.org/10.13140/2.1.2275.1046

[17] M. Pipattanasomporn, H. Feroze, and S. Rahman, "Multiagent systems in a distributed smart grid: Design and implementation." IEEE, Mar. 2009, pp. 1-8. [Online]. Available: http://ieeexplore.ieee.org/lpdocs/epic03/wrapper.htm?arnumber=4840087

[18] S. A. Deloach, M. F. Wood, and C. H. Sparkman, "MULTIAGENT SYSTEMS ENGINEERING," International Journal of Software Engineering and Knowledge Engineering, vol. 11, no. 03, pp. 231-258, Jun. 2001. [Online]. Available: http://www.worldscientific.com/doi/abs/10.1142/S0218194001000542

[19] K. H. Dam and M. Winikoff, "Comparing Agent-Oriented Methodologies," in Agent-Oriented Information Systems, T. Kanade, J. Kittler, J. M. Kleinberg, F. Mattern, J. C. Mitchell, O. Nierstrasz, C. Pandu Rangan, B. Steffen, M. Sudan, D. Terzopoulos, D. Tygar, M. Y. Vardi, G. Weikum, P. Giorgini, B. Henderson-Sellers, and M. Winikoff, Eds. Berlin, Heidelberg: Springer Berlin Heidelberg, 2004, vol. 3030, pp. 78-93. [Online]. Available: http://link.springer.com/10.1007/978-3540-25943-5 6

[20] P. Moraitis and N. Spanoudakis, "THE GAIA2jade PROCESS FOR MULTI-AGENT SYSTEMS DEVELOPMENT," Applied Artificial Intelligence, vol. 20, no. 2-4, pp. 251-273, Feb. 2006. [Online]. Available: http://www.tandfonline.com/doi/abs/10.1080/08839510500484249

[21] R. Neruda, "Ontology Description of Jade Computational Agents in OWL-DL." IEEE, 2010, pp. 514-518. [Online]. Available: http://ieeexplore.ieee.org/lpdocs/epic03/wrapper.htm?arnumber=5474446

[22] O. Kazi and R. Neruda, "Ontological modeling of meta learning multiagent systems in OWL-DL," IAENG International Journal of Computer Science, vol. 39, no. 4, pp. 357-362, Dec. 2012.

[23] R. Girardi and A. N. Lindoso, "An Ontology-Driven Technique for the Architectural and Detailed Design of Multi-agent Frameworks," in Agent-Oriented Information Systems III. Berlin, Heidelberg: Springer Berlin Heidelberg, 2006, vol. 3529, pp. 124-139. [Online]. Available: http://link.springer.com/10.1007/11916291_9

[24] I. I. Bittencourt, P. Bispo, E. Costa, J. Pedro, D. Vras, D. Dermeval, and H. Pacca, "Modeling JADE Agents from GAIA Methodology under the Perspective of Semantic Web," in Enterprise Information Systems. Berlin, Heidelberg: Springer Berlin Heidelberg, 2009, vol. 24, pp. 780-789. [Online]. Available: http://link.springer.com/10.1007/978-3642-01347-8_65

[25] S. Howell, Y. Rezgui, J.-L. Hippolyte, and M. Mourshed, "Semantic Interoperability for Holonic Energy Optimization of Connected Smart Homes and Distributed Energy Resources," Limoassol, Cyprus, Sep. 2016, paper accepted, May 2016.

[26] V. tuikys, M. Montvila, and R. Damaeviius, "Development of WEB Component Generators Using One-Stage Metaprogramming," Information Technology And Control, vol. 38, no. 2, pp. 108-118, 2009.

[27] F. Bellifemine, A. Poggi, and G. Rimassa, "JADE A FIPA-compliant agent framework," in Proceedings of the Fourth International Conference on Practical Application of Intelligent Agents and Multi-Agents Technology, London, Apr. 1999. 